\title{
Does Enjoyment in Life Improve the Lipid Profile of Caregivers?
}

\author{
Akemi Hirano ${ }^{1, *}$, Yusuke Suzuki ${ }^{2}$, Toshio Hayashi ${ }^{3}$, Koichiro Ina ${ }^{4}$, Joji Onishi ${ }^{5}$ \\ ${ }^{1}$ Department of Adult Nursing, Shubun University, 6 Nikko-cho, Ichinomiya, Aichi 491-0938, Japan. \\ ${ }^{2}$ Centre for Community Liaison and Patient Consultations, Nagoya University Hospital, 65 Tsuruma, Showa, Nagoya, Aichi \\ 466-8550, Japan. \\ ${ }^{3}$ Department of Community and In-Home Nursing, School of Health Sciences, Nagoya University Graduate School of Medicine, \\ Daiko-Minami, Higashi-ku, Nagoya, Aichi 461-8673, Japan. \\ ${ }^{4}$ Department of Internal Medicine, Ina Clinic, 3-111 Hirabari, Tenpaku, Nagoya, Aichi 468-0011, Japan. \\ ${ }^{5}$ Department of Community Healthcare \& Geriatrics, Nagoya University Graduate School of Medicine, 65 Tsuruma, Showa, Nagoya, \\ Aichi 466-8550, Japan.
}

How to cite this paper: Akemi Hirano, Yusuke Suzuki, Toshio Hayashi, Koichiro Ina, Joji Onishi. (2021) Does Enjoyment in Life Improve the Lipid Profile of Caregivers? International Journal of Clinical and Experimental Medicine Research, 5(4), 514-520.

DOI: 10.26855/ijcemr.2021.10.014

Received: September 8, 2021

Accepted: October 3, 2021

Published: November 2, 2021

*Corresponding author: Akemi Hirano, Department of Adult Nursing, Shubun University, 6 Nikko-cho, Ichinomiya, Aichi 491-0938, Japan.

Email: hirano@med.nagoya-u.ac.jp

\begin{abstract}
Background: Alzheimer caregivers have an increased fibrin turnover as compared to non-caregiving controls. Many intervention studies have aimed to reduce mental health disorders in caregivers, with little emphasis on physical changes and disease risk. Caregivers are prone to cardiovascular disease due to chronic stress, but few studies have examined their lipid levels. The present study aimed to examine whether having enjoyment in life can improve the lipid profile of caregivers. Methods: This intervention study targeted caregivers of patients with dementia aged $\geq 65$ years. Participants were divided into the intervention group and control group; those in the former group were asked to implement enjoyment in their lives, while those in the latter group continued with their normal lives. The intervention period was 24 weeks. Statistical analysis was performed using two-way analysis of variance. Results: Tests of within-participant effects revealed a significant interaction between triglyceride (TG) and sex [F $(1.64,62.45)=7.36, p=0.003]$, but no significant interaction was observed for HDL-C or LDL-C. Tests of within-participant comparisons revealed significant interactions between LDL-C and group $\mathrm{x}$ sex $(\mathrm{P}=0.040)$, and between TG and sex $(\mathrm{P}=0.02)$, but no significant interaction was observed for HDL-C. Tests of between-participant effects revealed a significant interaction between HDL-C and sex $(\mathrm{P}=0.001)$, but no significant interaction was observed for LDL-C or TG. Pairwise comparisons revealed no significant differences in HDL, LDL, and TG. Discussion: Analyses of the lipid profile of caregivers revealed sex differences in lipid levels. These differences may be related to caregiving burden and lifestyle changes due to unfamiliar caregiving tasks in male caregivers, in addition to the influence of dyslipidemia and progression of cardiovascular disease by sex. Worsening of the lipid profile may be due to chronic stress. The reason for improved HDL-C levels in the control group might be explained by changes in lifestyle. Lifestyle characteristics of caregivers need to be clarified in the future. Conclusion: Our findings suggest that the quality of life of caregivers can be improved by conducting further research that takes into account the sex of caregivers, and by clarifying factors that worsen their health status.
\end{abstract}




\section{Keywords}

Dementia, Caregiver, Enjoyment, Lipid

\section{Introduction}

The average coronary heart disease risk score is higher in caregivers than in non-caregivers [1]. Long-term caregivers have a significantly impaired cardiovagal baroreflex sensitivity relative to non-caregivers [2]. Alzheimer caregivers have an increased fibrin turnover as compared to non-caregiving controls [3]. The stress of caregiving leads to a loss of lymphocyte beta(2)-adrenergic receptors in more vulnerable caregivers [4]. Duration of caregiving is positively associated with intima-media thickness (IMT) measured in the internal/bifurcation segments of the carotid artery [5]. Caregivers have a higher frequency of carotid plaques compared with non-caregivers [6].

High caregiver burden has been reported to be a factor that affects the health of caregivers [7]. Dementia caregivers have more stress and physical problems than non-dementia caregivers [8]. Elderly caregivers with stressful care conditions and metabolic syndrome are at risk for declining health [9]. Frequent comorbidities experienced after becoming caregivers of dementia patients include hypertension [10]. Stress reportedly lowers HDL [11]. Therefore, it is necessary to alleviate the stress of caregiving.

A number of intervention studies have been conducted in caregivers of dementia patients. One study reported that a high level of trait mindfulness may have a protective effect on blood pressure when caregivers of dementia face high levels of stress [12]. In another study, greater engagement in pleasant leisure activities was associated with reduced mean arterial blood pressure [13]. The odds of the need for more respite is reportedly 5.3 times higher for family caregivers of people with dementia compared to family caregivers of people with musculoskeletal conditions, and 7.7 times higher compared to family caregivers of people with circulatory conditions [14].

According to previous intervention studies, programs with high levels of physical activity are not likely to be feasible as caregivers are already physically fatigued from caregiving responsibilities. Therefore, different methods of intervention are needed, for example, encouraging participants to find enjoyment in their daily lives, which can be easily and continuously implemented.

Internet-based self-management interventions demonstrated promising preliminary effects on caregiver self-efficacy and goal attainment [15]. The effectiveness of Internet-based interventions has been reported, including a study showing that caregivers in the experimental group had significantly reduced symptoms of depression and anxiety post-intervention [16]. While many studies targeting caregivers have aimed to reduce mental health disorders, few intervention studies have focused on the risk of diseases in this population. Moreover, few studies have examined the lipid profile of caregivers, despite the high likelihood of cardiovascular disease in caregivers due to chronic stress. Accordingly, the present study aimed to examine whether having enjoyment in life can improve the lipid profile of caregivers.

\section{Methods}

This intervention study targeted caregivers of patients with dementia aged $\geq 65$ years. Caregivers who had fun in their lives were excluded. Caregivers with cardiovascular disease were also excluded, but those receiving regular treatment for the disease and who had well-controlled hypertension, diabetes, and dyslipidemia were included.

Participants were divided into the following two groups: the intervention group, in which participants were asked to implement things to look forward to in their lives, and the control group, in which participants were asked to continue with their normal lives. The intervention period was 24 weeks.

Changes in high-density lipoprotein cholesterol (HDL-C), low-density lipoprotein cholesterol (LDL-C), and triglyceride (TG) were examined as factors that cause atherosclerosis, which affects the cardiovascular system. Two-way analysis of variance was performed, with the significance level set at less than 5\%. SPSS software was used for all analyses.

The Ethics Committee of Nagoya University Graduate School of Medicine approved this study. All participants provided written informed consent. Appropriate ethical measures were taken, such as clarifying that participation was voluntary. 


\section{Results}

Both the intervention and control groups consisted of 21 participants, with no significant difference in characteristics between the two groups (Table 1). Male participants accounted for $47.6 \%$ and $33.3 \%$ in the intervention and control groups, respectively, and $47.6 \%$ and $33.3 \%$ of participants were aged 65-74 years, respectively. At baseline, HDL-C and LDL-C were higher in females compared to males, and TG was higher in males compared to females, in both the intervention and control groups.

Table 2 shows the results of tests of within-participant effects using two-way repeated measures analysis of variance. No significant interaction was observed between HDL-C and group (intervention/control) $(\mathrm{P}=0.709)$, sex $(\mathrm{P}=0.218)$, and group $\mathrm{x}$ sex $[\mathrm{F}(1.69,64.39)=0.39, \mathrm{p}=0.644]$. Multiple comparisons using the Bonferroni test revealed no significant difference between the intervention and control groups $(\mathrm{P}=0.738)$. For LDL-C, no significant interaction was observed with group (intervention/control) $(\mathrm{P}=0.521)$, sex $(\mathrm{P}=0.233)$, or group x sex [F (2.0, 76.0) $=2.76, \mathrm{p}=0.070]$. Multiple comparisons using the Bonferroni test revealed no significant difference between the intervention and control groups $(\mathrm{P}=0.178)$. For $\mathrm{TG}$, no significant interaction was observed with group $(\mathrm{P}=0.399)$ or group $\mathrm{x} \operatorname{sex}(\mathrm{P}=0.299)$, whereas a significant interaction was observed with sex $[\mathrm{F}(1.64,62.45)=7.36$, $\mathrm{p}=0.003]$. Multiple comparisons using the Bonferroni test revealed no significant difference between the intervention and control groups $(\mathrm{P}=0.36)$, or by sex $(\mathrm{P}=0.58)$.

Table 3 shows the results of tests of within-participant comparisons. For HDL-C, no significant interaction was observed with group $(\mathrm{P}=0.629)$, sex $(\mathrm{P}=0.860)$, or group $\mathrm{x}$ sex $(\mathrm{P}=0.351)$. For LDL-C, no significant interaction was observed with group $(\mathrm{P}=0.832)$ or sex $(\mathrm{P}=0.075)$, whereas a significant interaction was observed with group $\mathrm{x}$ sex $(\mathrm{P}=0.040)$. For TG, a significant interaction was observed with sex $(\mathrm{P}=0.02)$, but not with group $(\mathrm{P}=0.43)$ or group $\mathrm{x} \operatorname{sex}(\mathrm{P}=0.53)$.

Table 4 shows the results of tests of between-participant effects. For HDL-C, a significant interaction was observed with sex $(\mathrm{P}=0.001)$. However, no significant interaction was observed with group $(\mathrm{P}=0.738)$ or group $\mathrm{x}$ sex $(\mathrm{P}=0.904)$. For LDL-C, no significant interaction was observed with group $(\mathrm{P}=0.18)$, sex $(\mathrm{P}=0.10)$, or group $\mathrm{x}$ sex $(\mathrm{P}=0.48)$. For $\mathrm{TG}$ as well, no significant interaction was observed with group $(\mathrm{P}=0.36)$, sex $(\mathrm{P}=0.58)$, or group $\mathrm{x}$ $\operatorname{sex}(\mathrm{P}=0.71)$.

Pairwise comparisons revealed no significant differences in HDL-C, LDL-C, and TG (Table 5).

Tables 6 show rates of change in the intervention and control groups. In the intervention group, rates of change were $-0.35 \%$ for HDL-C, $+0.17 \%$ for LDL-C, and $+14.6 \%$ for TG. In the control group, rates of change were $+1.77 \%$ for HDL-C, $+0.46 \%$ for LDL-C, and $+6.12 \%$ for TG.

Table 1. Characteristics of study participants

\begin{tabular}{ccccccccc}
\hline & \multicolumn{3}{c}{ Intervention group } & \multicolumn{3}{c}{ Control group } \\
& Mean & \pm & SD & Mean & \pm & SD & \\
\hline Age 65-74 years (N/\%) & 10 & $/$ & 47.6 & 7 & $/$ & 33.3 & \\
$\geq 75$ years (N/\%) & 11 & $/$ & 52.4 & 14 & & 66.7 & \\
HDL-C (standard value: $40-96 \mathrm{mg} / \mathrm{dL})$ & 56.6 & \pm & 15.3 & 56.4 & \pm & 15.9 & 0.605 \\
LDL-C (standard value: $70-139 \mathrm{mg} / \mathrm{dL})$ & 116.1 & \pm & 20.7 & 108.2 & \pm & 24.5 & 0.240 \\
TG (standard value: $50-149 \mathrm{mg} / \mathrm{dL})$ & 117.1 & \pm & 47.3 & 129.0 & \pm & 56.1 & 0.239 \\
\hline
\end{tabular}

Notes: HDL-C: high-density lipoprotein cholesterol; LDL-C: low-density lipoprotein cholesterol; TG: triglyceride.

Table 2. Results of tests of within-participant effects

\begin{tabular}{ccccc}
\hline & Source & Degree of freedom & F value & $P$ \\
\hline \multirow{2}{*}{ HDL-C } & HDL-C x Group* Sex & 1.69 & 0.39 & 0.64 \\
& Error & 64.39 & 2.76 & 0.07 \\
LDL-C & LDL-C x Group* Sex & 2.00 & & \\
& Error & 76.00 & 7.36 & 0.003 \\
TG & TG x Sex & 1.64 & 1.21 & 0.299 \\
& TG x Group*Sex & 1.64 & 62.45 & \\
\hline
\end{tabular}

Notes: Group refers to either the intervention or control group. HDL-C: high-density lipoprotein cholesterol; LDL-C: low-density lipoprotein cholesterol; TG: triglyceride. 
Table 3. Results of tests of within-participant comparisons

\begin{tabular}{ccccc}
\hline & Source & Degree of freedom & F value & $P$ \\
\hline \multirow{2}{*}{ HDL-C } & HDL-C x Group*Sex & 1 & 0.89 & 0.351 \\
& Error & 38 & 4.51 & 0.04 \\
LDL-C & LDL-C x Group* Sex & 1 & & \\
& Error & 38 & 6.42 & 0.02 \\
TG & TG x Sex & 1 & 0.40 & 0.53 \\
& TG x Group*Sex & 1 & & \\
\hline
\end{tabular}

Notes: Group refers to either the intervention or control group. HDL-C: high-density lipoprotein cholesterol; LDL-C: low-density lipoprotein cholesterol; TG: triglyceride.

Table 4. Tests of between-participant effects

\begin{tabular}{ccccc}
\hline & Source & Degree of freedom & F value & $P$ \\
\hline \multirow{2}{*}{ HDL-C } & Sex & 1 & 12.73 & 0.001 \\
& Group*Sex & 1 & 0.014 & 0.904 \\
LDL-C & Error & 38 & 0.51 & 0.481 \\
& Group*Sex & 1 & & 0.31 \\
TG & Error & 38 & 0.14 & 0.58 \\
& Sex & 1 & 1 & 0.71 \\
\hline
\end{tabular}

Notes: HDL-C: high-density lipoprotein cholesterol; LDL-C: low-density lipoprotein cholesterol; TG: triglyceride.

Table 5. Pairwise comparisons

\begin{tabular}{cccc}
\hline & Variable & Difference in mean values & $P$ \\
\hline HDL-C & Group & 1.49 & 0.74 \\
LDL-C & Group & 9.67 & 0.18 \\
TG & Group & 15.71 & 0.36 \\
\hline
\end{tabular}

Notes: Group: Intervention group, Control group. HDL-C: high-density lipoprotein cholesterol; LDL-C: low-density lipoprotein cholesterol; TG: triglyceride.

Table 6. Results of comparisons

\begin{tabular}{ccccc}
\hline & Intervention group & & Control group & \\
& Mean & Rate of change (\%) & Mean & Rate of change (\%) \\
\hline HDL-C & Pre56.6 $>$ Post56.4 & -0.35 & Pre56.4 $<$ Post57.4 & +1.77 \\
LDL-C & Pre116.1 $<$ Post116.3 & +0.17 & Pre108.2 $<$ Post108.7 & +0.46 \\
TG & Pre117.1 $<$ Post134.2 & +14.6 & Pre129.0 $<$ Post136.9 & +6.12 \\
\hline
\end{tabular}

Notes: HDL-C: high-density lipoprotein cholesterol; LDL-C: low-density lipoprotein cholesterol; TG: triglyceride. Rate of change: Rate of change after intervention $(\%)=($ Post-intervention value - baseline value $) /$ baseline value x 100.

\section{Discussion}

This study examined whether having enjoyment in life would lead to changes in the lipid profile, an indicator of health status, of caregivers. There was a significant interaction between sex and lipid profile. A significant group interaction was also observed for LDL-C. Pairwise comparisons revealed no significant differences in HDL-C, LDL-C, and TG. On the other hand, the rate of change of HDL-C showed a significant improvement in the control group. The rate of change of LDL-C in the control group was worse than that in the intervention group, whereas the rate of change of TG in the intervention group was worse than that in the control group.

Caregiving may have a bigger impact on TG than other lipids, and this may explain why caregivers have in- 
creased cardiometabolic risk [17]. LDL-C and TG concentrations are lower in women than in men aged $\leq 50$ years [18]. Women have a higher incidence of cardiovascular disease after menopause [19]. In addition, dyslipidemia is a risk factor for cardiovascular disease in older adults [20].

A previous study reported that male caregivers had higher TG and lower HDL-C levels compared to male non-caregivers [21]. In terms of sex differences, HDL-C levels have been reported to be higher in women than in men, and biochemical cardiovascular risk factors are lower in women than in men [22]. In the present study as well, male caregivers had lower HDL-C levels compared to female caregivers. In a previous study, male caregivers had elevated D-dimer and IL-6 levels, which are biomarkers of increased thrombosis and inflammation risk, respectively [23]. Moreover, male caregivers had increased diastolic blood pressure, higher blood glucose levels, lower hemoglobin and albumin levels, and slightly elevated TNF $\alpha$ and hs-CRP levels, both of which are inflammatory biomarkers [24]. Male sex is also a risk factor for CVD morbidity and mortality [25], and male caregivers reportedly have more comorbidities than females [26]. Consistent with these previous findings, male participants in the present study tended to have a worse lipid profile than female participants. In addition to the impact of dyslipidemia and progression of cardiovascular disease due to sex, these findings may be related to caregiving burden and lifestyle changes in male caregivers, possibly due to unfamiliar caregiving tasks. Caregiving is a potential cause of stress for male caregivers, and the worsening of their lipid profile may have been influenced by chronic stress.

A number of studies that examined sex differences in caregiving reported higher levels of depressive symptoms and a higher risk for clinical depression in female caregivers compared to male caregivers [27]. Chronic depression is one of the most important cardiovascular risk factors for poor prognosis in patients with myocardial infarction [28]. As a factor, we can predict that the cause of depression is involved. The development of caregiver depression in many cases is associated with dementia-related behaviors [29]. Caregiver depression has been reported to increase the use of emergency departments by patients with dementia [30]. Accordingly, early response to depression in caregivers is necessary.

Previous studies on the health status of dementia caregivers have generally considered poor sleep status to be a factor that worsens the physical condition of caregivers. For example, sleep quality was found to be related to mental distress in dementia caregivers [31]. However, the present study did not investigate sleep quality. It will be important to investigate the lives of caregivers in the future to improve their quality of life.

In the pairwise comparisons, no significant differences were observed in HDC-L, LDL-C, and TG. However, their rates of change varied slightly between the intervention and control groups. While the present study aimed to examine the impact of enjoyment in caregivers' lives, the lipid profile of participants in the intervention group did not show a significant improvement, possibly because our intervention did not result in significant changes in activity levels. The reason why HDL-C improved in the control group is unclear, but it is possible that there were some changes in lifestyles. A future study will be needed to further clarify the characteristics of caregiver lifestyles.

\section{Conclusion}

In this study, analyses of the lipid profile of caregivers revealed sex differences. Currently, few reports have been published on the characteristics of mental burden and lifestyles of male caregivers and their physical effects. Our findings suggest the need to promote research that takes into account sex differences among caregivers. In addition, given that poor sleep quality is a factor that worsens health conditions, the quality of life can be improved by investigating the lifestyle of caregivers.

\section{Conflict of interest statement}

None to report.

\section{Acknowledgements}

We thank the participants for their cooperation.

\section{Author's contributions}

Akemi Hirano conceived the idea and designed the study. Akemi Hirano carried out the data analysis and interpretation. Akemi Hirano wrote the first draft of the manuscript and Yusuke Suzuki, Toshio Hayashi, Koichiro Ina, Joji Onishi critically discussed all versions of the manuscript. Yusuke Suzuki, Toshio Hayashi, and Koichiro Ina recruited the participants, and Akemi Hirano contributed to the overall supervision of the study. 


\section{References}

[1] von Känel, R., Mausbach, B. T., Patterson, T. L., Dimsdale, J. E., Aschbacher, K., Mills, P. J., Ziegler, M. G., Ancoli-Israel, S., Grant, I. (2008). Increased Framingham Coronary Heart Disease Risk Score in dementia caregivers relative to non-caregiving controls. Gerontology, 54(3): 131-137. doi: 10.1159/000113649. PMID: 18204247.

[2] Wu, K. K., Bos, T., Mausbach, B. T., Milic, M., Ziegler, M. G., von Känel, R., Allison, M. A., Dimsdale, J. E., Mills, P. J., Ancoli-Israel, S., Patterson, T. L., Grant, I. (2017). Long-term caregiving is associated with impaired cardiovagal baroreflex. J Psychosom Res., 103: 29-33. doi: 10.1016/j.jpsychores.2017.10.001. PMID: 29167045; PMCID: PMC5726529.

[3] von Kanel, R., Dimsdale, J. E., Adler, K. A., Patterson, T. L., Mills, P. J., Grant, I. (2005). Exaggerated plasma fibrin formation (D-dimer) in elderly Alzheimer caregivers as compared to noncaregiving controls. Gerontology, 51(1): 7-13. doi: 10.1159/000081428. PMID: 15591750.

[4] Mills, P. J., Adler, K. A., Dimsdale, J. E., Perez, C. J., Ziegler, M. G., Ancoli-Israel, S., Patterson, T. L., Grant, I. (2004). Vulnerable caregivers of Alzheimer disease patients have a deficit in beta 2-adrenergic receptor sensitivity and density. Am J Geriatr Psychiatry, 12(3): 281-286. PMID: 15126229.

[5] Roepke, S. K., Allison, M., Von Känel, R., Mausbach, B. T., Chattillion, E. A., Harmell, A. L., Patterson, T. L., Dimsdale, J. E., Mills, P. J., Ziegler, M. G., Ancoli-Israel, S., Grant, I. (2012). Relationship between chronic stress and carotid intima-media thickness (IMT) in elderly Alzheimer's disease caregivers. Stress, 15(2): 121-129. doi: 10.3109/10253890.2011.596866. PMID: 21790484; PMCID: PMC3223262.

[6] Roepke, S. K., Chattillion, E. A., von Känel, R., Allison, M., Ziegler, M. G., Dimsdale, J. E., Mills, P. J., Patterson, T. L., Ancoli-Israel, S., Calleran, S., Harmell, A. L., Grant, I. (2011). Carotid plaque in Alzheimer caregivers and the role of sympathoadrenal arousal. Psychosom Med., 73(2): 206-213. doi: 10.1097/PSY.0b013e3182081004. PMID: 21217096; PMCID: PMC3077060.

[7] Oldenkamp, M., Hagedoorn, M., Slaets, J., Stolk, R., Wittek, R., Smidt, N. (2016). Subjective burden among spousal and adult-child informal caregivers of older adults: results from a longitudinal cohort study. BMC Geriatr, 16(1): 208. doi: 10.1186/s12877-016-0387-y. PMID: 27923347; PMCID: PMC5142272.

[8] Pinquart, M., Sörensen, S. (2003). Differences between caregivers and noncaregivers in psychological health and physical health: a meta-analysis. Psychol Aging, 18(2): 250-267. doi: 10.1037/0882-7974.18.2.250. PMID: 12825775.

[9] Fredman, L., Doros, G., Cauley, J. A., Hillier, T. A., Hochberg, M. C. (2010). Caregiving, metabolic syndrome indicators, and 1-year decline in walking speed: results of Caregiver-SOF. J Gerontol A Biol Sci Med Sci., 65(5): 565-572. doi: 10.1093/gerona/glq025. PMID: 20351074; PMCID: PMC2854884.

[10] Montgomery, W., Goren, A., Kahle-Wrobleski, K., Nakamura, T., Ueda, K. (2018). Alzheimer’s disease severity and its association with patient and caregiver quality of life in Japan: results of a community-based survey. BMC Geriatr, 18(1): 141. doi: 10.1186/s12877-018-0831-2. PMID: 29898679; PMCID: PMC6000944.

[11] Gowey, M. A., Khodneva, Y., Tison, S. E., Carson, A. P., Cherrington, A. L., Howard, V. J., Safford, M. M., Dutton, G. R. (2019). Depressive symptoms, perceived stress, and metabolic health: The REGARDS study. Int J Obes (Lond), 43(3): 615-632. doi: 10.1038/s41366-018-0270-3. PMID: 30518827; PMCID: PMC6405306.

[12] Vara-García, C., Romero-Moreno, R., Márquez-González, M., Mausbach, B. T., von Känel, R., Gallego-Alberto, L., Olmos, R., Losada, A. (2019). Stress and Blood Pressure in Dementia Caregivers: The Moderator Role of Mindfulness. Clin Gerontol, 42(5): 512-520. doi: 10.1080/07317115.2018.1554611. PMID: 30560734.

[13] Mausbach, B. T., Romero-Moreno, R., Bos, T., von Känel, R., Ziegler, M. G., Allison, M. A., Mills, P. J., Dimsdale, J. E., Ancoli-Israel, S., Losada, A., Márquez-González, M., Patterson, T. L., Grant, I. (2017). Engagement in Pleasant Leisure Activities and Blood Pressure: A 5-Year Longitudinal Study in Alzheimer Caregivers. Psychosom Med, 79(7): 735-741. doi: 10.1097/PSY.0000000000000497. PMID: 28640179; PMCID: PMC5573635.

[14] Vecchio, N., Fitzgerald, J. A., Radford, K., Kurrle, S. (2018). Respite service use among caregivers of older people: comparative analysis of family dementia caregivers with musculoskeletal and circulatory system disorder caregivers. Aging Ment Health, 22(1): 92-99. doi: 10.1080/13607863.2016.1232368. PMID: 27661453.

[15] Boots, L. M., de Vugt, M. E., Withagen, H. E., Kempen, G. I., Verhey, F. R. (2016). Development and Initial Evaluation of the Web-Based Self-Management Program "Partner in Balance" for Family Caregivers of People With Early Stage Dementia: An Exploratory Mixed-Methods Study. JMIR Res Protoc., 5(1): e33. doi: 10.2196/resprot.5142. PMID: 26932438; PMCID: PMC4795319.

[16] Blom, M. M., Zarit, S. H., Groot Zwaaftink, R. B., Cuijpers, P., Pot, A. M. (2015). Effectiveness of an Internet intervention for family caregivers of people with dementia: results of a randomized controlled trial. PLoS One, 10(2): e0116622. doi: 10.1371/journal.pone.0116622. PMID: 25679228; PMCID: PMC4334515.

[17] Ross, A., Shamburek, R., Wehrlen, L., Klagholz, S. D., Yang, L., Stoops, E., Flynn, S. L., Remaley, A. T., Pacak, K., Shelburne, N., Bevans, M. F. (2017). Cardiometabolic risk factors and health behaviors in family caregivers. PLoS One, 12(5): e0176408. doi: 10.1371/journal.pone.0176408. PMID: 28472106; PMCID: PMC5417518. 
[18] Matthan, N. R., Jalbert, S. M., Barrett, P. H., Dolnikowski, G. G., Schaefer, E. J., Lichtenstein, A. H. (2008). Gender-specific differences in the kinetics of nonfasting TRL, IDL, and LDL apolipoprotein B-100 in men and premenopausal women. Arterioscler Thromb Vasc Biol, 1838-1843. doi: 10.1161/ATVBAHA.108.163931. PMID: 18658047; PMCID: PMC2872098.

[19] Ley, S. H., Li, Y., Tobias, D. K., Manson, J. E., Rosner, B., Hu, F. B., Rexrode, K. M. (2017). Duration of Reproductive Life Span, Age at Menarche, and Age at Menopause Are Associated With Risk of Cardiovascular Disease in Women. J Am Heart Assoc., 6(11): e006713. doi: 10.1161/JAHA.117.006713. PMID: 29097389; PMCID: PMC5721766.

[20] van Bussel, E. F., Hoevenaar-Blom, M. P., Poortvliet, R. K. E., Gussekloo, J., van Dalen, J. W., van Gool, W. A., Richard, E., Moll van Charante, E. P. (2020). Predictive value of traditional risk factors for cardiovascular disease in older people: A systematic review. Prev Med., 132:105986. doi: 10.1016/j.ypmed.2020.105986. PMID: 31958478.

[21] Vitaliano, P. P., Russo, J., Niaura, R. (1995). Plasma lipids and their relationships with psychosocial factors in older adults. J Gerontol B Psychol Sci Soc Sci., 50(1): 18-24. doi: 10.1093/geronb/50b.1.p18. PMID: 7757819.

[22] Raberin, A., Connes, P., Barthélémy, J. C., Robert, P., Celle, S., Hupin, D., Faes, C., Rytz, C., Roche, F., Pialoux, V. (2020). Role of Gender and Physical Activity Level on Cardiovascular Risk Factors and Biomarkers of Oxidative Stress in the Elderly. Oxid Med Cell Longev., 1315471. doi: 10.1155/2020/1315471. PMID: 32655757; PMCID: PMC7321518.

[23] Mills, P. J., Ancoli-Israel, S., von Känel, R., Mausbach, B. T., Aschbacher, K., Patterson, T. L., Ziegler, M. G., Dimsdale, J. E., Grant, I. (2009). Effects of gender and dementia severity on Alzheimer's disease caregivers' sleep and biomarkers of coagulation and inflammation. Brain Behav Immun., 23(5): 605-610. doi: 10.1016/j.bbi.2008.09.014. PMID: 18930805; PMCID: PMC2757046.

[24] Stacey, A. F., Gill, T. K., Price, K., Taylor, A. W. (2019). Biomedical health profiles of unpaid family carers in an urban population in South Australia. PLoS One, 14(3): e0208434. doi: 10.1371/journal.pone.0208434. PMID: 30921333; PMCID: PMC6438668.

[25] van Bussel, E. F., Richard, E., Busschers, W. B., Steyerberg, E. W., van Gool, W. A., Moll van Charante, E. P., Hoevenaar-Blom, M. P. (2019). A cardiovascular risk prediction model for older people: Development and validation in a primary care population. J Clin Hypertens (Greenwich), 21(8): 1145-1152. doi: 10.1111/jch.13617. PMID: 31294917; PMCID: PMC6772108.

[26] Pöysti, M. M., Laakkonen, M. L., Strandberg, T., Savikko, N., Tilvis, R. S., Eloniemi-Sulkava, U., Pitkälä, K. H. (2012). Gender differences in dementia spousal caregiving. Int J Alzheimers Dis., 162960. doi: 10.1155/2012/162960. PMID: 23056990; PMCID: PMC3465980.

[27] Alfakhri, A. S., Alshudukhi, A. W., Alqahtani, A. A., Alhumaid, A. M., Alhathlol, O. A., Almojali, A. I., Alotaibi, M. A., Alaqeel, M. K. (2018). Depression Among Caregivers of Patients With Dementia. Inquiry, 55: 46958017750432. doi: 10.1177/0046958017750432. PMID: 29345180; PMCID: PMC5798670.

[28] De Hert, M., Detraux, J., Vancampfort, D. (2018). The intriguing relationship between coronary heart disease and mental disorders. Dialogues Clin Neurosci, 20(1): 31-40. doi: 10.31887/DCNS.2018.20.1/mdehert. PMID: 29946209; PMCID: PMC6016051.

[29] Choi, S. S. W., Budhathoki, C., Gitlin, L. N. (2019). Impact of three dementia-related behaviors on caregiver depression: The role of rejection of care, aggression, and agitation. Int J Geriatr Psychiatry, 34(7): 966-973. doi: 10.1002/gps.5097. PMID: 30897238; PMCID: PMC6579654.

[30] Guterman, E. L., Allen, I. E., Josephson, S. A., Merrilees, J. J., Dulaney, S., Chiong, W., Lee, K., Bonasera, S. J., Miller, B. L., Possin, K. L. (2019). Association Between Caregiver Depression and Emergency Department Use Among Patients With Dementia. JAMA Neurol, 76(10): 1166-1173. doi: 10.1001/jamaneurol.2019.1820. PMID: 31282955; PMCID: PMC6618767.

[31] Smyth, A., Whitehead, L., Quigley, E., Vafeas, C., Emery, L. (2020). Disrupted sleep and associated factors in Australian dementia caregivers: a cross-sectional study. BMC Geriatr, 20(1): 312. doi: 10.1186/s12877-020-01726-1. PMID: 32854627; PMCID: PMC7453710. 